Methods: Patients (11 male, 5 female, mean age $38.6 \pm 12.0$ years) with clinically active -axSpA (BASDAI>4, failure of NSAIDs, no previous biologics) prospectively underwent 3-Tesla and [18F]F PET/MRI (40 minutes after injection of a mean activity of $157 \mathrm{MBq}[18 \mathrm{~F}] \mathrm{F})$. Images of the SIJ ( $\mathrm{n}=16$ patients) and the whole spine ( $n=10$ patients) were performed at BL and FU. Three readers ( 1 for [18F]F/MRI and 2 for conventional MRI) evaluated all images independently and blinded to timepoint allocation. Only lesions on which all readers agreed on were used for further analyses. Inflammation (bone marrow edema, BME), structural lesions (fat deposition (FD), sclerosis, erosions and ankylosis) and focal [18F]F uptake were recorded on the level of SIJ (SIJ-Q) and vertebral quadrants (V-Q), with each SIJ or vertebral body consisting of 4 VQs (superior and inferior sacral and iliac for the SIJ, and superior and inferior, anterior and posterior for the vertebral bodies).

Results: A total of $128 \mathrm{SIJ}-\mathrm{Q}$ and 920 VQs were analyzed at both BL and FU. In the SIJs, 75 (58.6\%), 120 (93.8\%), 69 (53.9\%), 99 (77.3\%) and $16(12.5 \%)$ SIJ-Q showed BME, FD, sclerosis, erosions and ankylosis, while $111(86.7 \%)$ SIJ-Q showed focal [18F]F-uptake at BL. Association with increased [18F] F-uptake was found most frequently in SIJ-Q with BME (70/75 SIJ-Q, 93.3\%), sclerosis (65/69 SIJ-Q, 94.2\%) and FD (105/120 SIJ-Q, 87.5\%). At FU, 37 SIJ-Q still showed BME (improvement by $50.7 \%$ ), while almost no changes were observed in chronic lesions. In comparison, improvement of focal [18F] F-uptake was found in all lesion combinations, with improvement of focal [18F] F-lesions associated with BME by $62.9 \%$, with sclerosis by $33.8 \%$ and with FD by $22.9 \%$ of SIJ-Q.

In the spine, only 41 (4.5\%), $61(6.6 \%), 14(1.5 \%) \mathrm{V}-\mathrm{Q}$ showed BME, FD and sclerosis, respectively, while $77 \mathrm{~V}-\mathrm{Q}(8.4 \%)$ showed focal [18F]F-uptake. An association to increased [18F]F-uptake was found most frequently with sclerosis (7/14 V-Q, 50\%) and FD (25/61 V-Q, 41\%). At FU, 12 V-Q still showed BME (improvement by $70.7 \%$ ), while, similar to SIJ, almost no changes were observed in the chronic lesions. The largest improvement was found in focal [18F]F-lesions associated with BME $81.8 \%$ and with FD by $22.9 \%$ of $\mathrm{V}-\mathrm{Q}$.

Conclusion: In this first prospective study on whole spine and SIJ [18F]F/MRI in patients with r-axSpA, a significant decrease of osteoblastic activity was observed over 4 months of continuous anti-TNF treatment. The effect of treatment was observed not only at sites with inflammatory lesions (BME) but also at sites with pre-existing chronic structural lesions, while some osteoblastic activity remained visible at 4 months. These data support a short-term effect of anti-TNF treatment on osteoblastic activity, while the long-term effects need to be further studied. References:

[1] Van der Heijde D et al, Ann Rheum Dis 2017

[2] Buchbender C et al, J Rheumatol 2015

This work was supported by an unrestricted Grant by MSD GmbH, Germany Disclosure of Interests: Xenofon Baraliakos Grant/research support from: Grant/research support from: AbbVie, BMS, Celgene, Chugai, Merck, Novartis, Pfizer, UCB and Werfen, Consultant of: AbbVie, BMS, Celgene, Chugai, Merck, Novartis, Pfizer, UCB and Werfen, Speakers bureau: AbbVie, BMS, Celgene, Chugai, Merck, Novartis, Pfizer, UCB and Werfen, Styliani Tsiami: None declared, Christoph Rischpler: None declared, Nils-Martin Bruckmann: None declared, Wolfgang Fendler: None declared, Julian Kirchner: None declared, Ken Hermann: None declared, Lino Sawicki: None declared, Juergen Braun Grant/research support from: Abbvie (Abbott), Amgen, BMS, Boehringer, Celgene, Celltrion, Centocor, Chugai, Eli Lilly and Company, Medac, MSD (Schering Plough), Mundipharma, Novartis, Pfizer (Wyeth), Roche, Sanofi- Aventis, and UCB Pharma, Consultant of: Abbvie (Abbott), Amgen, BMS, Boehringer, Celgene, Celltrion, Centocor, Chugai, EBEWE Pharma, Eli Lilly and Company, Medac, MSD (Schering-Plough), Mundipharma, Novartis, Pfizer (Wyeth), Roche Sanofi-Aventis, and UCB Pharma, Speakers bureau: Abbvie (Abbott), Amgen, BMS, Boehringer, Celgene, Celltrion, Centocor, Chugai, EBEWE Pharma, El Lilly and Company, Medac, MSD (Schering-Plough), Mundipharma, Novartis Pfizer (Wyeth), Roche, Sanofi-Aventis, and UCB Pharma DOI: 10.1136/annrheumdis-2020-eular.5573

\section{SAT0366 \\ CLINICAL RESPONSE TO BIOLOGIC DMARDS IN AXIAL SPONDYLOARTHRITIS AND AXIAL PSORIATIC ARTHRITIS. DIFFERENT DISEASES, SAME OUTCOMES?}

D. Benavent ${ }^{1}$, C. Plasencia ${ }^{1}$, K. N. Franco Gomez ${ }^{1}$, L. Nuño ${ }^{1}$, A. Balsa ${ }^{1}$, V. Navarro-Compán ${ }^{1}{ }^{1}$ Hospital La Paz, Idipaz, Rheumatology, Madrid, Spain

Background: Patients with psoriatic arthritis may present predominant axial involvement. Currently, it is unclear whether these patients should be considered as axial spondyloarthritis (axSpA) with psoriasis or psoriatic arthritis with axial involvement -also known as axial PsA (axPsA). Data comparing medium-term treatment response to biological drugs in axSpA and axPsA would add relevant information to answer this question.

Objectives: To compare the clinical response and predictor factors after one year of biological therapy in patients with axSpA and axPsA.
Methods: One-year follow-up data from all patients (pts) with axSpA or axPsA (defined by the treating rheumatologist) included in a prospective cohort of pts receiving biological therapy from la Paz University Hospital between 2002 and 2019 were analysed. Demographic information, laboratory tests, concomitant treatments and disease status were collected at baseline. Clinical disease activity was measured by PhGA and ASDAS criteria at baseline, 6 and 12 months. According to ASDAS, disease activity was defined as: inactive disease (ID) (ASDAS <1.3) low disease activity (LDA) (ASDAS 1.3-2.1), high disease activity (HDA) (ASDAS 2.1-3.5) and very high disease activity (VHDA) (ASDAS >3.5). Clinical importan improvement and major improvement were defined by ASDAS (delta-ASDAS $\geq 1.1$ and $\geq 2.0$, respectively). According to PhGA, disease activity was assorted by consensus of 3 expert rheumatologists in: ID with PhGA $<5$, LDA with PhGA 5-30, HDA with PhGA $>30-60$ and VHDA with PhGA >60. Clinical improvement by PhGA was defined as an improvement of $30 \%$ compared to baseline. In the statistical analysis, the frequency of pts achieving each clinical activity status and clinical improvement at $6 \mathrm{~m}$ and $12 \mathrm{~m}$ were compared using Fisher test, separately for axSpA and axPsA Baseline predictor factors for achieving clinical response and clinical improvement were identified using univariable and multivariable binary regression.

Results: Out of 352 included pts, 287 (81.5\%) had axSpA and 65 (18.5\%) axPsA. Sixty percent were males, 158 (45\%) smokers, with mean (SD) baseline disease activity of ASDAS (bASDAS): 3.3 (0.9) and PhGA: 39.1 (21.5). Biological therapies initiated included TNF inhibitors in $93.8 \%$ and secukinumab in $6.2 \%$ In comparison to axPsA, pts with axSpA were more HLA B27 positive $(p<0.001)$ and had better PhGA at baseline $(p=0.02)$. They also had more uveitis $(p=0.03)$ and were more radiographically affected $(p<0.001)$.

Response rates at $6 \mathrm{~m}$ and $12 \mathrm{~m}$ in both diseases according to ASDAS are shown in Figure 1, and to PhGA in Figure 2. Both diseases presented a similar clinical response, and no statistically significant differences were observed for any disease activity interval between them for ASDAS or PhGA. There were no differ ences between both diseases on clinical improvement, regardless the type of measurement.

$\underline{\%}$

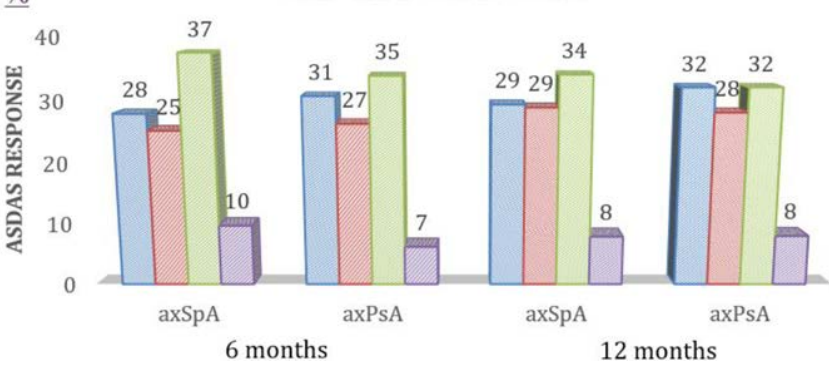

Figure 1. Response rates (in percentage) by ASDAS at $6 \mathrm{~m}$ and $12 \mathrm{~m}$ in axSpA and PsA

In the group of axSpA, the univariate analysis observed that LDA (by ASDAS) at $12 \mathrm{~m}$ was associated with bASDAS $(\mathrm{OR}=0.67, \mathrm{p}=0.02)$, male gender $(\mathrm{OR}=2.8$, $\mathrm{p}=0.001)$ and HLA B27 positive (OR=2.3, $p=0.01)$. In the multivariate analysis these variables remained significantly associated with LDA (bASDAS: OR $=0.67$ $p<0.05$; male gender: $O R=2.7, p<0.01$; and $H L A B 27$ positivity $O R=2.6, p<0.01$ ) In the group of axPsA, the univariate analysis showed a tendency that male pts achieved LDA more frequently at $6 \mathrm{~m}(\mathrm{OR}=3.0, \mathrm{p}=0.05)$ and at $12 \mathrm{~m}(\mathrm{OR}=2.75$ $\mathrm{p}=0.09$ ). In the multivariable analyses, none of the factors was significantly associated neither with clinical improvement nor with LDA in pts with axPsA.

Conclusion: In clinical practice, pts with axSpA and axPsA present a similar clinical response to biological therapy within the first year of treatment. Male pts seem to have better medium-term outcomes in both diseases, and HLA B27 pts respond better in axSpA.

Disclosure of Interests: Diego Benavent: None declared, Chamaida Plasencia: None declared, Karen Nathalie Franco Gomez: None declared, Laura Nuño: None declared, Alejandro Balsa Grant/research support from: BMS, Roche, Consultant of: AbbVie, Gilead, Lilly, Pfizer, UCB, Sanofi, Sandoz, Speakers bureau: AbbVie, Lilly, Sanofi, Novartis, Pfizer, UCB, Roche, Nordic, Sandoz, Victoria Navarro-Compán Consultant of: Abbvie, Lilly, Novartis, Pfizer, UCB, Speakers bureau: AbbVie, MSD, Lilly, Novartis, Pfizer, UCB

DOI: 10.1136/annrheumdis-2020-eular.3095

\section{SAT0367 \\ EXTRA-ARTICULAR MANIFESTATIONS IN EARLY AXIAL SPONDYLOARTHRITIS: WHAT IS THEIR FREQUENCY? A SYSTEMATIC LITERATURE REVIEW} INCLUDING 2854 PATIENTS

E. Bilgin ${ }^{1}$, U. Kalyoncu', L. Gossec ${ }^{2} .{ }^{1}$ Hacettepe University, Ankara, Turkey; ${ }^{2}$ Sorbonne Universite, Paris, France 
Background: Extra-articular manifestations (EAMs): psoriasis, uveitis and inflammatory bowel disease (IBD) are common in patients with established spondyloarthritis (SpA), with prevalences reported around $9 \%, 26 \%$ and $7 \%$ respectively (1). However, data on the prevalence of EAMs are lacking in early axial SpA (axSpA). Objectives: The aim was to assess the prevalence of EAMs in early axSpA in the published literature.

Methods: Systematic literature search on Pubmed MEDLINE up to 31.12.2019 with keywords referring to EAMs (uveitis and synonyms, psoriasis and synonyms or IBD and synonyms) and early axSpA (recent, young adult, young, untreated, inception) and selection by one reader of all full-text publications in English, describing the prevalence of at least one of the EAMs in patients with early axSpA, defined here as patients fulfilling ASAS, ESSG or Amor criteria and symptom duration of less than 6 years (as this was defined by authors as early disease). Patients' age, axSpA symptom duration, sex, HLA-B27 status, and number of patients with EAMs were recorded by one reader using a predefined extraction sheet. For longitudinal studies, baseline data was recorded. Description of patients was analysed using weighted means. Prevalences in each study according to symptom duration were graphically reported, and pooled prevalences were calculated by meta-analysis of proportions, using a random-effects model and the DerSimonian \& Laird method to derive the summary estimate.

Results: Of 667 articles, 17 were relevant to the research question with prevalence data of psoriasis, uveitis and IBD available in 16, 17 and 15 articles, respectively (and most studies reporting several EAMs). Of the 17 articles, 14 were cohort studies and 3 were trials in early axSpA. A total of 2854 patients with early SpA was analyzed: weighted mean age 32.3 \pm 9.1 years (range 21-42 years), weighted mean axSpA symptom duration $20.7 \pm 11.1$ months (range 8-68), $40.3 \%$ were female, and $65.1 \%$ carried HLA-B27.

The pooled prevalences of psoriasis, uveitis and IBD were respectively $8.9 \%$ $(95 \% \mathrm{Cl} 5.0,13.8), 13.4 \%(95 \% \mathrm{Cl} 9.5,17.8)$ and $3.5 \%(95 \% \mathrm{Cl} 1.7,5.9)$ (Figure 1). There was a trend towards higher prevalences in patients with longer disease duration (Figure 2).

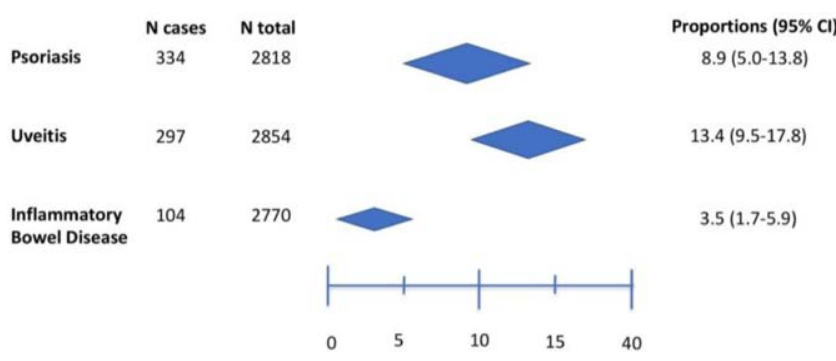

Figure 1. Meta-analysis of prevalence of each EAM

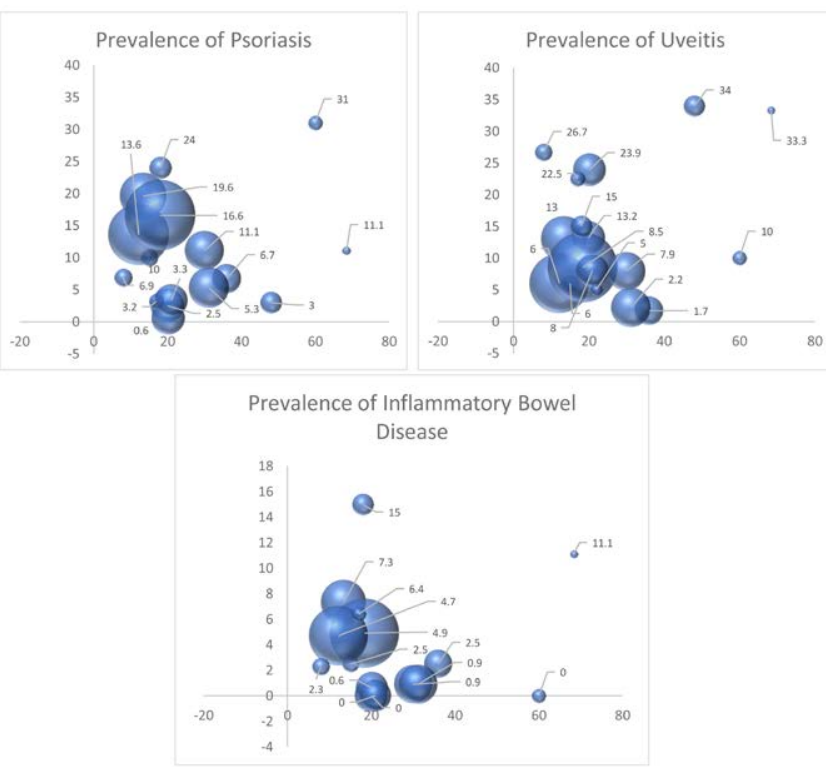

Figure 2. The prevalence of each EAM according to the symptom duration in early axSpAX axis: mean symptom duration (months). $Y$ axis: prevalence (\%) of an EAM. Diameter of bubbles is proportional to sample size of each article.

Conclusion: Over the first years of axSpA, EAMs are frequent, in particular psoriasis and uveitis, with prevalences up to $30 \%$ in some studies. Compared to established axSpA, the EAM which was much less frequent was uveitis, which suggests the appearance of new cases over follow-up. Physicians need to screen carefully for EAMs right from the time of diagnosis, and need to repeat this screening over follow-up.

\section{References:}

[1] Stolwijk C et al. Prevalence of extra-articular manifestations in patients with ankylosing spondylitis: a systematic review and meta-analysis. Ann Rheum Dis. 2015;74(1):65-73

Disclosure of Interests: Emre Bilgin: None declared, Umut Kalyoncu Consultant of: Abbvie, Amgen, Janssen, Lilly, Novartis, UCB, Laure Gossec Grant/research support from: Lilly, Mylan, Pfizer, Sandoz, Consultant of: AbbVie, Amgen, Biogen, Celgene, Janssen, Lilly, Novartis, Pfizer, Sandoz, Sanofi-Aventis, UCB DOI: 10.1136/annrheumdis-2020-eular.3067

\section{SAT0368 PREGNANCY IN WOMEN WITH SPONDYLOARTHRITIS: WHO ARE THE PATIENTS AT RISK OF DISEASE FLARE?}

F. Crisafulli ${ }^{1}$, A. R. Cruz-Machado ${ }^{2}$, M. C. Gerardi ${ }^{1}$, M. Filippini ${ }^{1}$, M. Fredi ${ }^{1}$, R. Gorla ${ }^{1}$, M. G. Lazzaroni ${ }^{1}$, C. Nalli ${ }^{1}$, M. Taglietti ${ }^{1}$, A. Lojacono ${ }^{3}$, C. Zanardini ${ }^{3}$, S. Zatti ${ }^{3}$, L. Andreoli ${ }^{1}$, F. Franceschini ${ }^{1}$, A. Tincani ${ }^{1} .{ }^{1}$ ASST Spedali Civili and University of Brescia, Rheumatology and Clinical Immunology Unit, Brescia, Italy; ${ }^{2}$ Hospital de Santa Maria, Serviço de Reumatologia e Doenças Ósseas Metabólicas, Lisboa, Portugal; ${ }^{3}$ ASST Spedali Civili and University of Brescia, Obstetrics and Gynaecology Unit, Brescia, Italy

Background: Patients with Spondyloarthritis (SpA) can experience flares during pregnancy and postpartum even though the available data are limited and not conclusive.

Objectives: To assess disease activity and treatment modification during pregnancy and postpartum in patients with SpA and to identify risk factors for disease flare.

Methods: Data on SpA pregnancies prospectively-followed in a pregnancy clinic from 2010 to 2019 were retrospectively analysed. Disease activity was assessed during each trimester and postpartum using ASDAS-CRP or DAS28-CRP. Flare was defined as an increase of disease activity leading to treatment modification (introduction or increase $\geq 5 \mathrm{mg} /$ day of prednisone, introduction of cDMARD or bDMARD) ${ }^{1}$

Results: Data on 50 pregnancies in 46 patients were collected (mean age at conception $33 \pm 4.7$ years; median disease duration: 60 months (IQR 24-132) 33 psoriatic arthritis, 6 axialSpA, 2 reactive arthritis, 2 IBD-related SpA; 6 undifferentiated $\mathrm{SpA}, 1$ juvenile idiopathic arthritis). Six pregnancies ended in miscarriage, so they weren't considered for the analysis of flares during pregnancy (table 1). Fifteen out of $44(34 \%)$ pregnancies had at least one flare during pregnancy $\left(6,7\right.$ and 4 during $1^{\text {st }}, 2^{\text {nd }}$ and $3^{\text {rd }}$ trimester respectively; 2 pregnancies had multiple flares). A higher rate of flare was observed in pregnancies of patients with axial involvement $(p=0.01)$, on treatment with bDMARDs at preconceptional visit $(\mathrm{p}=0.03)$ and who stopped TNFi at positive pregnancy test $(p=0.03)$. Peripheral involvement was associated with a lower rate of flares $(p=0.02)$. Medications resumed during pregnancy were steroids (in 6 pregnancies), cDMARDs (2 sulfasalazine, 1 cyclosporine) and bDMARDs (4 certolizumab, 4 etanercept). During postpartum period flares were recorded in $46 \%$ of patients.

Table 1. clinical features, medication and disease activity in pregnancies with flare vs without flare

\begin{tabular}{|c|c|c|c|}
\hline CLINICAL FEATURES & FLARE (15) & NO FLARE (29) & $p$ \\
\hline Axial involvement, $\mathrm{n}(\%)$ & $11 / 15(73)$ & $9 / 29(31)$ & 0.01 \\
\hline Peripheral arthritis, $n(\%)$ & 8/15 (53) & $26 / 29(90)$ & 0.02 \\
\hline Enthesitis, n (\%) & $5 / 15(33)$ & $14 / 29(48)$ & ns \\
\hline Dactilitis, n (\%) & $3 / 15(20)$ & $8 / 29(28)$ & ns \\
\hline Psoriasis, n (\%) & $6 / 15(40)$ & $17 / 29(59)$ & ns \\
\hline IBD, n (\%) & $2 / 15(13)$ & 0 & ns \\
\hline Uveitis, $n(\%)$ & $1 / 15(7)$ & $3 / 29(10)$ & ns \\
\hline $\mathrm{HLAB} 27+$ & $7 / 11(64)$ & $5 / 12(42)$ & ns \\
\hline \multicolumn{4}{|l|}{ MEDICATION HISTORY } \\
\hline bDMARDs, n (\%) & $11 / 15(73)$ & $7 / 29(24)$ & 0.003 \\
\hline bDMARDs at preconception visit, $\mathrm{n}(\%)$ & $8 / 15(53)$ & $6 / 29(21)$ & 0.04 \\
\hline bDMARDs stopped at positive pregnancy test, $\mathrm{n}(\%)$ & $7 / 15(47)$ & $4 / 29(14)$ & 0.03 \\
\hline cDMARDs, n (\%) & $12 / 15(80)$ & $25 / 29(86)$ & ns \\
\hline \multicolumn{4}{|l|}{ DISEASE ACTIVITY } \\
\hline ACTIVE DISEASE* preconception visit, $\mathrm{n}(\%)$ & $3 / 14(21)$ & $4 / 23(17)$ & ns \\
\hline ACTIVE DISEASE $1^{\text {st }}$ trimester, $\mathrm{n}(\%)$ & $6 / 15(40)$ & $1 / 29(3)$ & 0.004 \\
\hline ACTIVE DISEASE $2^{\text {nd }}$ trimester, $n(\%)$ & $8 / 15(47)$ & $2 / 29(7)$ & 0.001 \\
\hline ACTIVE DISEASE $3^{\text {rd }}$ trimester, $\mathrm{n}(\%)$ & 2/15 (13) & $1 / 29(3)$ & ns \\
\hline
\end{tabular}

*DAS28-CRP $>3.2$ or ASDAS-CRP $\geq 2.1$

Conclusion: In our cohort of prospectively-followed SpA pregnancies, 34\% experienced a flare during pregnancy and $46 \%$ during postpartum. Flares 